\title{
Can we Predict Molar-Incisor-Hypomineralisation from Hypomineralised Primary Dentition?: A Systematic Review and a Meta-Analysis
}

\author{
Wafa Hammami ${ }^{1 *}$ and Soumaya Touzi ${ }^{2}$ \\ ${ }^{1}$ Resident in the Department of Dental Anatomy and Pediatric Dentistry, Faculty of \\ Dentistry of Monastir, Tunisia \\ ${ }^{2}$ Professor in the Department of Dental Anatomy, Faculty of Dentistry of Monastir, \\ Tunisia
}

*Corresponding Author: Wafa Hammami, Resident in the Department of Dental Anatomy and Pediatric Dentistry, Faculty of Dentistry of Monastir, Tunisia.
Received: March 04, 2021

Published: March 26, 2021

(C) All rights are reserved by Wafa Hammami and Soumaya Touzi.

\begin{abstract}
Introduction: Molar-incisor-hypomineralisation (MIH) is a widespread lesion in the world. It usually occurs in the last stage of mineralisation of second primary molars; for this reason, a predictive relationship was assessed by several authors.

Objectives: The aim of this systematic review and meta-analysis was to investigate whether the occurrence of hypomineralised deciduous molars can predict molar-incisor-hypomineralisation lesions in children's permanent teeth.

Materials and Methods: A broad search in MedLine, Cochrane Library, Scopus, Web of Science, Ebscohost, Google scholar, Science direct and OpenGrey databases were conducted using all terms related to the subject. Manual search on grey literature was also performed. Relevant papers published in English since 2010 were identified after a review of their titles, abstracts and full text reading. Results: Eight references were selected and were eligible for the present systematic review and meta-analysis. A global Odds Ratio of 6.28 (95\% CI 3.45 - 11.41) (P< 0.01) was obtained and it confirmed the predictive relationship. The likelihood ratios proved it too. Conclusion: The presence of hypomineralised primary dentition especially second molars is a predictive indicator for MIH defect, with a greater prevalence for the mild form. Early detection of these defects and preventive measures are required to reduce MIH complications.
\end{abstract}

Keywords: Hypomineralisation; Deciduous Molars; Molar-Incisor-Hypomineralisation; Children

\section{Abbreviations}

MIH: Molar Incisor Hypomineralization; EAPD: European Academy of Paediatric Dentistry; PRISMA: Preferred Reporting Items for Systematic Reviews and Meta- Analyzes; PICOT: Participants, Interventions, Comparisons, Outcomes and Time; MeSH: Medical Subject Headings; HSPM: Hypomineralised Second Primary Molars; CASP: Critical Appraisal Skills Programme; RevMan: Review Manager; NOS: New Castle Ottawa; EAPD: European Academy of Paediatric Dentistry; ppm: Part Per Million; CPP-ACP: Casein Phosphopeptide-Amorphous Calcium Phosphate

\section{Introduction}

Molar incisor hypomineralization (MIH) is defined according to the European Academy of Paediatric Dentistry (EAPD) as a hypomineralisation of systemic origin of one to four permanent first molars frequently associated with affected incisors [1]. In the world, it 
highly rates in South and East Asia and High-income North America $[2,3]$.

The highest incident numbers in 2016 were found in South Asia, Southeast Asia, and Eastern Sub-Saharan Africa [2,3]. To date, the etiologies are widely varied and most of times unclear. Authors are convinced that it is a multifactorial lesion [4,5]. It causes teeth damage, discrimination, isolation in the societies [6] and emotional disorders in children especially at school level [7]. Thus MIH constitutes a public health problem [7]. Currently, the incapacity to highlight a deeming cause(s), makes risk's prevention actions difficult to undertake. In histology, ameloblasts showed the highest activities' amount in the first stage of mineralisation of the first permanent molars. It overlapped with the latest stage of calcification of second primary molars. Risk factors which appear during this period could affect them simultaneously $[8,9]$. That's why many authors investigate whether we can predict MIH from the hypomineralisation of deciduous molars, incisors and canines. IF there is a proven possibility of this association, preventive measures should be undertaken.

\section{Aim of the Study}

The aim of this systematic review and meta-analysis is to investigate whether the occurrence of hypomineralised deciduous molars can predict molar-incisor hypomineralisation lesions in children's permanent teeth.

\section{Materials and Methods}

This study is designed to review the literature regarding the relationship between hypomineralised lesions on primary teeth and molar incisor hypomineralisation.

It was conducted according to the Preferred Reporting Items for Systematic Reviews and Meta- Analyzes (PRISMA) criteria to improve the scientific credibility of the review. The acronym Participants, Interventions, Comparisons, Outcomes and Time (PICOT) was used to define the problem and ask the question that the study would like to answer on.

\section{Search strategy}

A detailed search between 2010 and 2019 was conducted by exploitation of several databases: Cochrane Library, Medline via Pubmed, Scopus, web of science, ebscohost, Science direct, Google scholar, OpenGrey.
A manual search on google was performed to investigate grey literature.

\section{Search terms}

The following terms were used as keywords or MeSH: 'Tooth', 'Deciduous', 'Molar', 'Incisor', 'Child', 'Hypomineralisation', 'Molar incisor hypomineralisation' and 'molar incisor hypomineralization'.

Boolean operators $<\mathrm{AND}>$ and $<\mathrm{OR}>$ were used to formulate the following search's equation: (Hypomineralized AND second AND primary AND molars) OR (deciduous AND molar AND hypomineralization) OR HSPM) OR ((molar AND incisor AND hypomineralization) OR (demarcated AND opacities) OR MIH).

\section{Inclusion criteria}

All studies that answer our research's question written in English. Meta-analysis, Systematic review, Cohort and Cross-sectional studies were considered to review.

\section{Non-inclusion criteria}

Poor scientific evidence based papers were not included: articles published before 2010, case reports, narrative studies (dissertations), clinical practice guidelines, thesis, chapters of textbooks or textbook, annals of congress, letter to the editor.

Data extraction and quality assessment

A pilot-tested spreadsheet for including articles was developed by the both reviewers The following items were considered relevant and thus collected: author, year, country, type of the publication, number of participants, age, type of dentition, criteria of examination, examined teeth, severity, co-occurrence of lesions.

The two reviewers independently extracted the necessary data using the form which has been tested and validated by the working group. In case of disagreement it was solved by a discussion. A kappa test for intra-examiners agreement was calculated. It was 0.7 which indicated a substantial understanding between them [10].

The quality assessments of the included studies and evaluated by the Preferred Reporting Items for Systematic Reviews and Meta-Analyses (PRISMA) checklist [11] and the Critical Appraisal Skills Programme (CASP) tool [12] then ranked with the Cochrane collaboration's tool for assessing risk bias. Low risk was in green, moderate in yellow and high risk in red (Figure 4). 


\section{Statistic}

Review Manager (RevMan) version 5.3 was manipulated to conduct the meta-analysis [13]. The odds ratio (OR) was calculated to test the association between hypomineralised second primary molars (HSPM) and MIH. Confidence interval (CI) was 95\% for all measures. The outcomes were obtained using random-effects models.

\section{Risk of bias}

The assessment of risk of bias for each article was performed with the New castle ottawa (NOS) criteria [14]. Observational studies give more strength to results than cross-sectional ones [15].

\section{Results}

Study selection

The electronic search identified a total of 881 articles and the hand searching provided 3 articles (Figure 1). After applying the language filter (English), the year of publication (2010 - 2019) and the elimination of duplicate all abstracts were assessed and those which do not meet the inclusion criteria were eliminated this step leads to the exclusion of 870 other articles. The full texts of the remaining articles were screened and those that did not meet the selection criteria were rejected ( 3 articles).
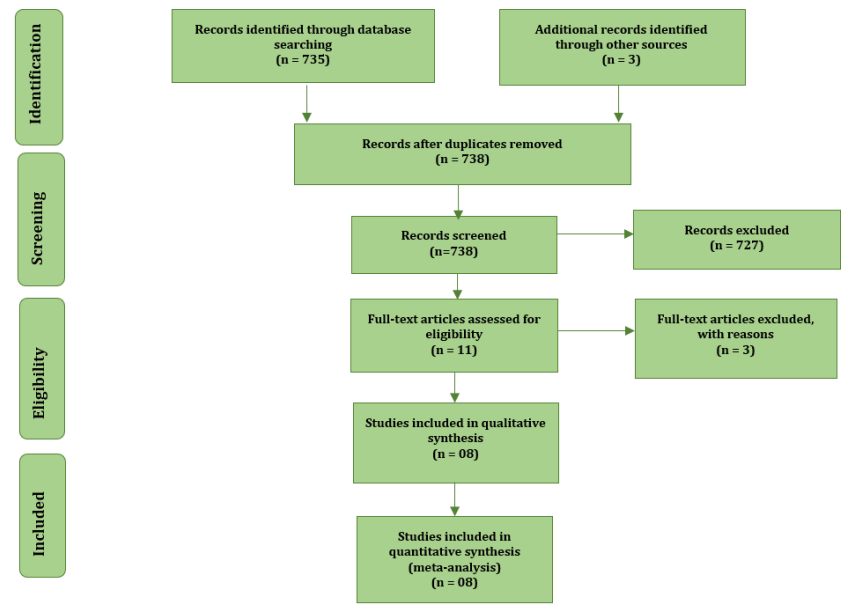

Figure 1: PRISMA flow diagram of the research of articles.

Finally, 8 articles were selected: 6 cross-sectional studies, 1 cohort study and 1 systematic review and meta-analysis (flow chart).
The meta-analysis was conducted with 6 articles. Studies with no statistics of Mittal R., et al. or old meta-analysis of Garot., et al. [16] were not included because they were not needed in the combination of results.

The studies were conducted in 5 different countries: 2 studies in Brazil, 2 in India, 1 in Spain, 1 in Netherlands and 1 in Iraq. Three studies were multicenter studies.

\section{Study characteristics}

In the selected studies sample size ranged from 134 to 5561 children. The total of participants was 6625 . Characteristics of the six studies are represented in table 1.

Articles were published between 2012 and 2017. Four studies were conducted in schools and two in medical centres. European Academy of Paediatric Dentistry (EAPD) diagnostic criteria for MIH were used in all of them. EAPD Criteria had also included absence or presence of demarcated opacities; post-eruptive enamel breakdown; atypical restorations and extraction of molars due to $\mathrm{MIH}$.

Five studies had performed intra-examiner reliability tests. It varied between 0.6 and 0.95 which mean perfect agreement [10].

For the risk of bias and according NCO scale, all studies showed at least five stars expect the study of Mittal.R which was a high area of biases.

Meta-analysis results

Homogeneity/heterogeneity

The forest plot showed that $\mathrm{X}^{2}$ test results in $19.24(\mathrm{P}=0.002)$ with degree of freedom $\mathrm{df}=5$ and alpha $5 \%$.

The low P-value $(\mathrm{P}=0.002)$ or the large chi-squared statistic relative to its degree of freedom $\left(X^{2}=19.24\right)$ provides evidence of heterogeneity (variation in effect estimates is beyond chance).

The values of Cochran's Q-value and P-value were $<0.1$ indicating statistically significant difference between studies [17]. This results is confirmed by I2 test which was equal to $74 \%$ (Figure 2).

A substantial heterogeneity across the studies exists [18].

No sub-group analysis could be performed due to the small number of studies. 


\begin{tabular}{|c|c|c|c|c|c|c|c|c|c|c|c|}
\hline \multirow[b]{2}{*}{ Study } & \multirow[b]{2}{*}{ type } & \multirow[b]{2}{*}{$\begin{array}{c}\text { Year of } \\
\text { publication }\end{array}$} & \multirow[b]{2}{*}{ Country } & \multirow{2}{*}{$\begin{array}{c}\text { Number of } \\
\text { partici- } \\
\text { pants }\end{array}$} & \multirow[b]{2}{*}{ Age } & \multirow{2}{*}{$\begin{array}{l}\text { Place of } \\
\text { examina- } \\
\text { tion }\end{array}$} & \multirow{2}{*}{$\begin{array}{c}\text { Criteria } \\
\text { of } \\
\text { diagnosis }\end{array}$} & \multirow{2}{*}{$\begin{array}{c}\text { Intra } \\
\text {-examiner } \\
\text { test }\end{array}$} & \multicolumn{3}{|c|}{ BIAS NOS Scale } \\
\hline & & & & & & & & & $\begin{array}{c}\text { Selection } \\
(* * * *)\end{array}$ & $\begin{array}{c}\text { Comparability } \\
(* *)\end{array}$ & $\begin{array}{l}\text { Outcome } \\
\left(* * * \text { or }^{* *}\right)\end{array}$ \\
\hline $\begin{array}{l}\text { Costa Silva } \\
\text { et al. }\end{array}$ & $\begin{array}{l}\text { Cross } \\
\text { sectional } \\
\text { study }\end{array}$ & 2014 & Brazil & 134 & $4-6$ & school & EAPD & - & $* * *$ & $* *$ & $* *$ \\
\hline $\begin{array}{l}\text { Figueiredo } \\
\text { Sé., et al. }\end{array}$ & $\begin{array}{l}\text { Cross } \\
\text { sectional } \\
\text { study }\end{array}$ & 2017 & Brazil & 1963 & $6-11$ & school & EAPD & 0.6 & * & $* *$ & $* *$ \\
\hline $\begin{array}{l}\text { Elfrink., } \\
\text { et al. }\end{array}$ & $\begin{array}{l}\text { Cohort } \\
\text { study }\end{array}$ & 2012 & $\begin{array}{c}\text { Nether- } \\
\text { lands }\end{array}$ & 6161 & $5-6$ & $\begin{array}{l}\text { Medical } \\
\text { center }\end{array}$ & EAPD & 0.95 & $* * *$ & $* *$ & $* * *$ \\
\hline $\begin{array}{l}\text { Ghanim., } \\
\text { et al. }\end{array}$ & $\begin{array}{l}\text { Cross } \\
\text { sectional } \\
\text { study }\end{array}$ & 2013 & Iraq & 809 & $7-9$ & School & EAPD & 0.8 & $* * *$ & $* *$ & $* *$ \\
\hline $\begin{array}{l}\text { Mittal.N., } \\
\text { et al. }\end{array}$ & $\begin{array}{l}\text { Cross } \\
\text { sectional } \\
\text { study } \\
\end{array}$ & 2015 & India & 978 & $6-8$ & school & EAPD & 0.92 & $* * *$ & $* *$ & $* *$ \\
\hline $\begin{array}{l}\text { Negre } \\
\text { Barber., } \\
\text { et al. }\end{array}$ & $\begin{array}{l}\text { Cross } \\
\text { sectional } \\
\text { study }\end{array}$ & 2016 & Spain & 414 & $8-9$ & $\begin{array}{l}\text { Medical } \\
\text { center }\end{array}$ & EAPD & 0.83 & $* * *$ & $* *$ & $* *$ \\
\hline $\begin{array}{l}\text { Mittal R., } \\
\text { et al. }\end{array}$ & $\begin{array}{l}\text { Cross } \\
\text { sectional } \\
\text { study }\end{array}$ & 2016 & India & 1109 & $3-12$ & school & EAPD & 0.88 & $*$ & $* *$ & - \\
\hline
\end{tabular}

Table 1: Included studies characteristics.

The use of the random-effects or fixed-effects model depends on the presence of statistical heterogeneity.

That 's why the meta-analysis was performed with a random model.

Odds ratio was 6.28 (95\% CI 3.45 - 11.41) ( $<$ < 0.01) (Figure 2).

\section{Weight of studies}

The weight of the co-occurrence of HSPM and MIH prevalence ranges between $11.9 \%$ and $19 \%$ (Figure 2). That's mean that studies offer valuable information and are almost closer to the 'true effect' we are trying to estimate [19].

\section{Sensitivity and specificity}

The forest plot for diagnostic test shows the means and the confidence intervals for sensitivity/specificity.

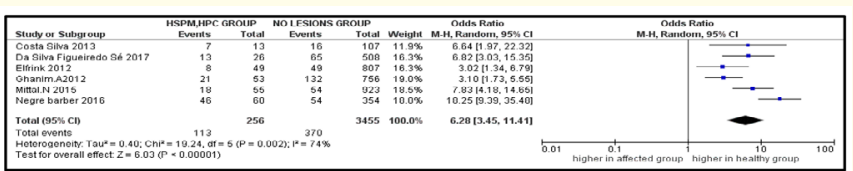

Figure 2: Forest plot of the association between HSPM and MIH by mean of odds ratio, I2 and degree of freedom.

All studies showed high specificity and low sensitivity too (Figure 3).

\section{Risk of bias}

To assess risk of bias data from article were entered in Revman 5.3 and graph was obtained showing the implication of each article in each type of biases. 


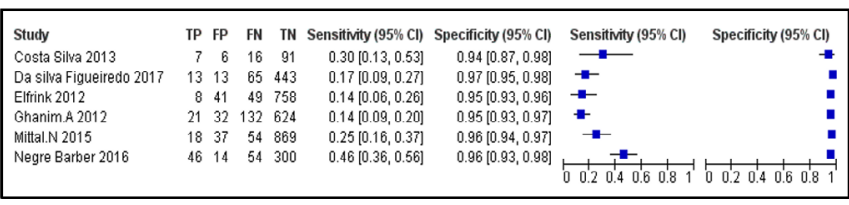

Figure 3: Forest plot of specificity and sensitivity.

The detection biases were present in Mittal R., et al.'s study [20], Ghanim A., et al.'s study [21] and in Figueiredo Sé., et al.'s study [22].

The selection biases were in Costa-Silva., et al.'s study 23 and in in Mittal R., et al.'s study [20]. Other biases can be cited in Ghanim A., et al.'s study [21] such as confounding bias of post eruptive enamel broken lesions due to hypomineralisation or caries (Figure 4).

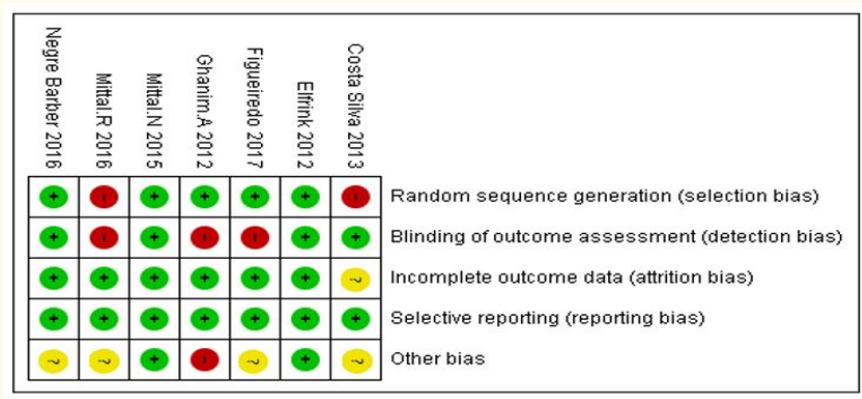

Figure 4: Risk of bias summary.

Positive likelihood ratio (PLR): sensitivity/(1-specificity) and Negative likelihood ratio (NLR): 1- sensitivity/specificity were calculated for clinical applicability (Figure 5). PLR was high for all studies and NLR was low.

\section{Discussion}

The effective sample size is formed by a total of 6625 included participants.

Results from the six included studies showed an OR $=6.28(95 \%$ CI 3.45 - 11.41) $(\mathrm{P}<0.01)$ which means a high link between HSPM and MIH.

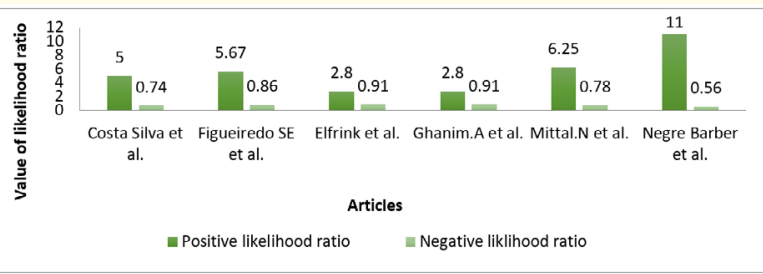

Figure 5: Value of likelihood of different included articles.

A distribution of effects exists, resulting in heterogeneity among the studies.

A random effects model was used, because, the fixed effect model was not appropriate when there was statistical heterogeneity [24]. The studies were weighted with the inverse of their variance to minimize the variation of the weighted average.

This review is including few studies that's why interpretation should be attentive.

Some sample size were small. Elfrink., et al. recommended 300 participants for prevalence studies [25] which would exclude the Costa-Silva., et al.'s study [23]. Costa-Silva., et al. did not show any association between HSPM and MIH (OR 2.12, 95\% CI 0.77 - 5.83) [23].

This lack would strengthen then the positive predictive relationship [23]. But we did not exclude studies based on sample size. we just highlight that it may influence the outcome. However, Ghanim., et al. [21] with 809 participants also did not find an association (OR 1.48, 95\% CI 0.79 - 2.77).

Thus, clinical heterogeneity seems to be clear.

Statistical heterogeneity of the included studies was considered high according to the $\mathrm{I}^{2}$ test (74\%) (Figure 2).

This diference can be due to the variation of effective samples size varying from 134 to 2327 participants or to the age range selection (3 to 12 years old) [5,8,14,25,20,22,26] (Table 1).

Subgroup analysis can not be done because the number of included studies is small. 
Diagnostic test showed low Sensitivity and high specificity for all studies [5,8,14,25,20,22,26] (Figure 3).

Low sensitivity can be explained by a low percentage of kids who had MIH had HSPM too. The high specificity is because children without MIH did not present HSPM too. Thus, false positives are low which confirm the alteration (MIH).

The High positive likelihood ratio ranging between 2.8 to 11; which means a powerful predictive indicator for the presence of $\mathrm{MIH}$, and the low negative likelihood ratio (0.56 to 0.91) (Figure 5) shows meant a low capacity to predict the absence of MIH in the children without HSPM.

In observational study, the assessment of a relationship between hypomineralisation in primary dentition and molar-incisor-hypomineralisation can be hidden by bias: selection bias, confounding factors and measurement error.

For the risk of bias considering selection biases, sample size should be representative. Study with low number of participants can under-estimate the prevalence of defects.

In Elfrink., et al.'s study sample size of children was with minimum number of 300 for prevalence and 1000 for aetiologic studies [25]. Costa-Silva., et al. [23] had noticed that there was limitation in their study about sample size.

For the age of children, there was a wide heterogeneity in studies age's range.

For hypomineralised primary teeth, the best age to assess them is 5 years old because all primary teeth should have been erupted and children are more cooperant than earlier age [25].

For permanent dentition, the EAPD recommended that the best age to assess the presence of MIH is 8 years old [25]. That is because, at this age, most of permanent first molar and incisors exist. An early age can under-estimate the prevalence: some teeth cannot be present (not yet erupted). A later age can overestimate the prevalence of MIH because other defects such as caries can appear.

The authors' choice of the age group of 6 - 7 years to investigate MIH $[8,14,25]$ may be explained by avoiding caries, alteration and masticatory forces. The Severity of hpomineralisation can change by external factor.
The variation in methods can influence the prevalence's results. For example, there were differences in light's tool. Some articles describe the use of natural light $[20,25]$ which varied with weather state. It can cause an underestimation of the prevalence of hypomineralisation if daily light decreases. Other studies claim the use of artificial light [5,8,14,22,26].

Photographs' evaluation by Elfrink [8] can be not considered as a bias. The sensitivity and specificity of scoring HSPM from intraoral photographs was tested and validated for epidemiological studies [27].

For Confounding biases, all included articles had referred to the EAPD 2003 criteria for MIH and DMH /HPC scoring with some modifications. There were articles which considered sound defect if diameter was $<1 \mathrm{~mm}[5,8,25]$ and other if $<2 \mathrm{~mm}[14,20,21]$. Moreover, there was a difference in defects definition. Some atypical fillings or caries can be classified with the post eruptive enamel breakdown lesions. Thus, these differences generate bias in results. So, the use of similar criteria should be done to allow a comparability between studies as stated Elfrink., et al. in 2015 [25].

Other limits in the studies can be noticed, such as absence of intra-examiner testing in one study [23]. Moreover, no information about blinding of participants and personnel was present. We recommend for future clinical studies an obligation of training the examiners, precision in evaluation criteria, and a follow-up period in order to reduce bias risk. Ghanim., et al. proposed recently a clinical trial protocol [28]. Diagnosis of enamel defects must be strengthened by quantitative methods to accurate diagnosis. Considering the graph obtained after meta-analysis conducting; the detection biases which concern the outcome were present in:

- Mittal R., et al.'s study [20] due to absence of statistic calcul.

- In Ghanim A et al.'s study [21] which stated a poor Odds ratio not demonstrated with table and calcul.

- And in Figueiredo Sé., et al.'s study [22] using Fisher test for corelation which do not prove a cause/effect relationship. The selection bias was the highest in Costa-Silva., et al.'s study [23] probably due to the limited sample size and in Ghanim A., et al. study [21] due to younger age selection which can overestimates the prevalence. Other biases can be stated in Mittal R., et al.'s paper [20] such as confunding bias. Post-eruptive enamel breakdown can be due to hypomineralisation or caries (Figure 4). 
Research question's response

After conducting the meta-analysis, we can ensure that there is a strong link between hypomineralisation in primary dentition and molar incisor hypomineralisation.

Articles which are opposed to this fact present limitation:

- A higher age range for Ghanim., et al.'s study [21] can overestimate the results.

- A low one for Costa-Silva., et al. [23] can underestimate it.

Severity of lesions in primary dentition seems to influence the presence of MIH in the permanent dentition. Authors explain that with mineralisation process. Mild lesion happened in the last stage of mineralisation of deciduous molars which is concomitant to first stage of mineralisation of the first permanent molar with a high activity of ameloblasts [9].

That's why a higher probability of developing molar incisor hypomineralisation was in children with mild hypomineralised second deciduous molars [9].

\section{Recommendations}

Preventive measures from the primary dentition should be undertaken to avoid complications. By enlightening patients parents to consult at early statue and regularly, managing this anomaly at an early age we can reduce its impact.

Indeed, prevention should start since hypomineralised teeth had erupted because they are more vulnerable to breakdown and carious lesion development [28].

Dietary advice for children's healthy life should be given.

Fluoride toothpastes with minimum of 1450 part per million (ppm) should be prescribed to reduce risk of caries and hypersensitivity.

CPP-ACP (casein phosphopeptide-amorphous calcium phosphate) in creme or sugar-free chewing gum to mineralize, desensitize and afford bio-available calcium and phosphate for the hypomineralised teeth were reported in in vitro [29] and in vivo studies [26]. Sealants using glass-ionomer cement for enamel loss and sealant with resin-based for not broken hypomineralised molars are used. Fluoride varnishes for sensitivity is applicated [28].

\section{Conclusion}

The presence of hypomineralised primary dentition especially second molars is a predictive factor for MIH defect, with greater prevalence for the mild form. Early detection of defects and preventive measures are required to reduce MIH complications.

\section{Competing Financial Interests}

The authors declare no competing financial interests.

\section{Conflicts of Interest}

The authors declare no conflicts of interest.

\section{Bibliography}

1. Weerheijm KL. "Molar incisor hypomineralisation (MIH)". European Journal of Paediatric Dentistry 4.3 (2003): 114-120.

2. Schwendicke F., et al. "Global burden of molar incisor hypomineralization". Journal of Dentistry 68 (2018): 10-18.

3. Schwendicke F., et al. "Corrigendum to "Global burden of molar incisor hypomineralization". Journal of Dentistry 80 (2019): 89-92.

4. Weerheijm KL. "Molar incisor hypomineralization (MIH): clinical presentation, aetiology and management". Dent Update 31.1 (2004): 9-12.

5. Silva MJ., et al. "Etiology of molar incisor hypomineralization - A systematic review". Community Dentistry and Oral Epidemiology 44.4 (2016): 342-353.

6. Scheffel DL., et al. "Esthetic dental anomalies as motive for bullying in schoolchildren". European Journal of Dentistry 8.1 (2014): 124-128.

7. Leal SC., et al. "Do parents and children perceive molar-incisor hypomineralization as an oral health problem?" International Journal of Paediatric Dentistry 27.5 (2017): 372-379.

8. Elfrink ME., et al. "Deciduous molar hypomineralization and molar incisor hypomineralization". Journal of Dental Research 91.6 (2012): 551-555.

9. Mittal N and Sharma BB. "Hypomineralised second primary molars: prevalence, defect characteristics and possible association with Molar Incisor Hypomineralisation in Indian chil- 
dren". European Archives of Paediatric Dentistry 16.6 (2015): 441-447.

10. Landis JR and Koch GG. "The measurement of observer agreement for categorical data". Biometrics 33.1 (1977): 159-174.

11. Preferred Reporting Items for Systematic Reviews and MetaAnalyses (2019).

12. Critical appraisal Skills Programme (CASP) (2019).

13. The Nordic Cochrane Centre. RevMan 5.3 User Guide (2018).

14. Wells GA., et al. "The Newcastle-Ottawa scale (NOS) for assessing the quailty of nonrandomised studies in meta-analyses (2019).

15. Weber C., et al. "Evidence base clinical management and utilisation of new technology in European neurosurgery". Acta Neurochirurgica 155.4 (2013): 747-754.

16. Garot E., et al. "Are hypomineralised lesions on second primary molars (HSPM) a predictive sign of molar incisor hypomineralisation (MIH)? A systematic review and a meta-analysis". Journal of Dentistry 72 (2018): 8-13.

17. Imamura M., et al. "Evidence-Based Urology Working Group. Evidence-based urology in practice: heterogeneity in a systematic review meta-analysis". BJU International 105.6 (2010): $770-773$.

18. Guyatt GH., et al. "Rating the quality of evidence--inconsistency”. Journal of Clinical Epidemiology 64.12 (2011): 1294-1302.

19. Akobeng AK. "Understanding systematic reviews and metaanalysis". Archives of Disease in Childhood 90.8 (2005): 845848.

20. Mittal R., et al. "Assessment of association between molar incisor hypomineralization and hypomineralized second primary molar". Journal of International Society of Preventive and Community Dentistry 6.1 (2016): 34-39.

21. Ghanim A., et al. "Prevalence of demarcated hypomineralisation defects in second primary molars in Iraqi children". International Journal of Paediatric Dentistry 23.1 (2013): 48-55.

22. Da Silva Figueiredo Sé MJ., et al. "Are Hypomineralized Primary Molars and Canines Associated with Molar-Incisor Hypomin- eralization?" Pediatric Dentistry 39.7 (2017): 445-449.

23. Costa-Silva CM., et al. "Influence of deciduous molar hypomineralization on the development of molar-incisor hypomineralizarion". Brazilian Journal of Oral Sciences 12.4 (2013): 335338.

24. Hak T., et al. "How to interpret results of meta-analysis (2019).

25. Elfrink ME., et al. "Standardised studies on Molar Incisor Hypomineralisation (MIH) and Hypomineralised Second Primary Molars (HSPM): a need". European Archives of Paediatric Dentistry 16.3 (2015): 247-255.

26. Luchini C., et al. "Assessing the quality of studies in metaanalyses: advantages and limitations of the Newcastle Ottawa Scale”. World Journal of Meta-Analysis 5.4 (2017): 80-84.

27. Elfrink ME., et al. "Validity of scoring caries and primary molar hypomineralization (DMH) on intraoral photographs". European Archives of Paediatric Dentistry 10 (2009): 5-10.

28. Ghanim A., et al. "Molar incisor hypomineralisation (MIH) training manual for clinical field surveys and practice". European Archives of Paediatric Dentistry 18.4 (2017): 225-242.

29. Zhou C., et al. "Casein phosphopeptide-amorphous calcium phosphate remineralization of primary teeth early enamel lesions". Journal of Dentistry 42.1 (2014): 21-29.

\section{Assets from publication with us}

- Prompt Acknowledgement after receiving the article

- Thorough Double blinded peer review

- Rapid Publication

- Issue of Publication Certificate

- High visibility of your Published work

Website: www.actascientific.com/

Submit Article: www.actascientific.com/submission.php

Email us: editor@actascientific.com

Contact us: +919182824667 